\title{
On Commutators of Fuzzy Multigroups
}

\author{
P. A. Ejegwa* and J. M. Agbetayo
}

\begin{abstract}
Fuzzy multigroup is an application of fuzzy multiset to group theory. Although, a lots have been done on the theory of fuzzy multigroups, some group's theoretic notions could still be investigated in fuzzy multigroup context. Certainly, the idea of commutator is one of such group's theoretic notions yet to be studied in the environment of fuzzy multigroups. Hence, the aim of this article is to establish the notion of commutator in fuzzy multigroup setting. A number of some related results are obtained and characterized. Among several results that are obtained, it is established that, if $A$ and $B$ are fuzzy submultigroups of a fuzzy multigroup $C$, then $[A, B] \subseteq A \cup B$ holds. Some homomorphic properties of commutator in fuzzy multigroup context are discussed. The notion of admissible fuzzy submultisets $A$ and $B$ of $C \in F M G(X)$ under an operator domain $\mathcal{D}$ is explicated, and it is shown that $(A, B)$ and $[A, B]$ are $\mathcal{D}$-admissible.
\end{abstract}

\section{Introduction}

Fuzzy set theory proposed by Zadeh [26] (although with heated disagreement as at then) has been widely studied with applications ranging from engineering and computer science to medical diagnosis and social behavior, etc. In a way of extending the application of fuzzy sets to group theory, Rosenfeld [22] proposed the idea of fuzzy groups as an application of fuzzy sets to group theory and some number of results were obtained. Numerous studies have been carried out on some group theoretic notions in fuzzy group setting (cf. [1, 4, 13, 18, 19, 20]).

By synthesizing the concepts of fuzzy sets and multisets (cf. [14]), the idea of fuzzy multisets or fuzzy bags was proposed in [25] as a generalization of fuzzy sets

Received: February 27, 2020; Accepted: May 2, 2020

2010 Mathematics Subject Classification: 03E72, 08A72, 20N25.

Keywords and phrases: commutator, fuzzy multiset, fuzzy multigroup, fuzzy submultigroup.

${ }^{*}$ Corresponding author

Copyright (c) 2020 Authors 
in multiset framework. Many researches have been carried out on the theory of fuzzy multisets (cf. [3, 15, 11, 15, 16, 17, 24]). In recent times, the concept of fuzzy multigroups was introduced as an application of fuzzy multisets to group theory [23]. The ideas of abelian fuzzy multigroups and order of fuzzy multigroups have been studied with some results [2, 6], and the notions of center and centralizer in fuzzy multigroup context were established [6]. In the same vein, the concept of fuzzy multigroupoids was introduced and the idea of fuzzy submultigroups was explored with a number of results [7]. The concept of normal fuzzy submultigroups has been established and some of it properties were explicated [8]. In [9], the idea of homomorphism in the environment of fuzzy multigroups was defined and some homomorphic properties of fuzzy multigroups were elaborated. Some group analog concepts were established in fuzzy multigroup context, as seen in [10, 12]. The idea of fuzzy multigroups was extended to t-norms and some results were established [21].

The notion of commutator occupied a loft height in crisp group theory. Notwithstanding, this idea has not been investigated in fuzzy multigroup setting. Thus, an attempt to establish commutator in fuzzy multigroup setting is not only necessary but overdue. This constitutes the motivation of the article. In recap, this paper assay to introduce commutator in fuzzy multigroups with some analog results. The remaining part of this article is thus presented: Section 2 provides some preliminaries on fuzzy multisets and fuzzy multigroups. In Section 3 , the idea of commutator in fuzzy multigroups is proposed and some of its properties are discussed. Section 4 discusses some homomorphic properties of commutator in fuzzy multigroup context. Lastly, Section 5 concludes the paper and provides direction for future studies.

\section{Preliminaries}

Definition 2.1 ([25]). Assume $X$ is a set of elements. Then, a fuzzy bag/multiset $A$ drawn from $X$ is an object of the form

$$
A=\left\{\left\langle\frac{C M_{A}(x)}{x}\right\rangle \mid x \in X\right\}
$$


defined by a count membership function

$$
C M_{A}: X \rightarrow Q
$$

where $Q$ is the set of all crisp bags or multisets from the unit interval $I=[0,1]$ and

$$
C M_{A}(x)=\left\{\mu_{A}^{1}(x), \mu_{A}^{2}(x), \ldots, \mu_{A}^{n}(x)\right\},
$$

where $\mu_{A}^{1}(x), \mu_{A}^{2}(x), \ldots, \mu_{A}^{n}(x) \in[0,1]$ such that $\mu_{A}^{1}(x) \geq \mu_{A}^{2}(x) \geq \ldots \geq \mu_{A}^{n}(x)$.

From [24], a fuzzy multiset can also be characterized by a high-order function. In particular, a fuzzy multiset $A$ can be characterized by a function

$$
C M_{A}: X \rightarrow N^{I} \text { or } C M_{A}: X \rightarrow[0,1] \rightarrow N,
$$

where $I=[0,1]$ and $N=\mathbb{N} \cup\{0\}$.

We denote the set of all fuzzy multisets by $F M S(X)$.

Definition $2.2([15])$. Let $A$ and $B$ be fuzzy multisets of $X$. Then

(i) $A \subseteq B \Leftrightarrow C M_{A}(x) \leq C M_{B}(x) \forall x \in X$.

(ii) $A=B \Leftrightarrow C M_{A}(x)=C M_{B}(x) \forall x \in X$.

(iii) $A \subset B \Leftrightarrow A \subseteq B$ and $A \neq B$.

(iv) $A \cap B \Rightarrow C M_{A}(x) \wedge C M_{B}(x) \forall x \in X$.

(v) $A \cup B \Rightarrow C M_{A}(x) \vee C M_{B}(x) \forall x \in X$.

Note that $\bigwedge$ and $\bigvee$ denote minimum and maximum operations.

Definition 2.3 ([23]). Suppose $X$ is a group. Then, a fuzzy multiset $A$ of $X$ is called a fuzzy multigroup of $X$ if the following conditions are satisfied:

(i) $C M_{A}(x y) \geq C M_{A}(x) \wedge C M_{A}(y) \forall x, y \in X$,

(ii) $C M_{A}\left(x^{-1}\right) \geq C M_{A}(x) \forall x \in X$. 
By implication, a fuzzy multiset $A$ over $X$ is called a fuzzy multigroup of a group $X$ if

$$
C M_{A}\left(x y^{-1}\right) \geq C M_{A}(x) \wedge C M_{A}(y), \forall x, y \in X .
$$

It follows immediately from the definition that,

$$
C M_{A}(e) \geq C M_{A}(x) \forall x \in X,
$$

where $e$ is the identity element of $X$. The second condition above is strictly $C M_{A}\left(x^{-1}\right)=C M_{A}(x) \forall x \in X$, since $C M_{A}(x)=C M_{A}\left(\left(x^{-1}\right)^{-1}\right) \geq C M_{A}\left(x^{-1}\right)$.

We denote the set of all fuzzy multigroups of $X$ by $F M G(X)$.

Definition 2.4 ([8]). Let $A, B \in F M G(X)$. Then, the product $A \circ B$ is defined to be a fuzzy multiset of $X$ as follows:

$$
C M_{A \circ B}(x)= \begin{cases}\bigvee_{x=y z}\left[C M_{A}(y) \wedge C M_{B}(z)\right], & \text { if } \exists y, z \in X \text { such that } x=y z \\ 0, & \text { otherwise. }\end{cases}
$$

Proposition 2.5 ([23]). Let $A \in F M S(X)$. Then $A \in F M G(X)$ if and only if $A \circ A=A$.

Definition 2.6 ([7]). Let $A \in F M G(X)$. A fuzzy submultiset $B$ of $A$ is called a fuzzy submultigroup of $A$ denoted by $B \subseteq A$ if $B$ is a fuzzy multigroup. A fuzzy submultigroup $B$ of $A$ is a proper fuzzy submultigroup denoted by $B \subset A$, if $B \subseteq A$ and $A \neq B$.

Proposition 2.7 ([7]). Let $A \in F M G(X)$. Then, the sets $A_{*}$ and $A^{*}$ defined by

$$
A_{*}=\left\{x \in X \mid C M_{A}(x)>0\right\}
$$

and

$$
A^{*}=\left\{x \in X \mid C M_{A}(x)=C M_{A}(e)\right\}
$$

are subgroups of $X$.

Proposition 2.8. Let $A \in F M G(X)$. Then, the set $A_{[\alpha]}$ defined by

$$
A_{[\alpha]}=\left\{x \in X \mid C M_{A}(x) \geq \alpha, \alpha \in[0,1]\right\}
$$


is a subgroup of $X$ for $\alpha \leq C M_{A}(e)$ and $A^{[\alpha]}$ defined by

$$
A^{[\alpha]}=\left\{x \in X \mid C M_{A}(x)>\alpha, \alpha \in[0,1]\right\}
$$

is a subgroup of $X$ for $\alpha \geq C M_{A}(e)$.

Definition 2.9 ([8]). Let $A, B \in F M G(X)$ such that $A \subseteq B$. Then, $A$ is called a normal fuzzy submultigroup of $B$ if for all $x, y \in X$,

$$
C M_{A}\left(x y x^{-1}\right)=C M_{A}(y) .
$$

Definition 2.10 ([6]). Let $A \in F M G(X)$. Then, $A$ is said to be commutative if for all $x, y \in X$,

$$
C M_{A}(x y)=C M_{A}(y x) .
$$

Definition 2.11 ([9]). Let $X$ and $Y$ be groups and let $f: X \rightarrow Y$ be a homomorphism. Suppose $A$ and $B$ are fuzzy multigroups of $X$ and $Y$, respectively. Then, $f$ induces a homomorphism from $A$ to $B$ which satisfies

(i) $C M_{A}\left(f^{-1}\left(y_{1} y_{2}\right)\right) \geq C M_{A}\left(f^{-1}\left(y_{1}\right)\right) \wedge C M_{A}\left(f^{-1}\left(y_{2}\right)\right) \forall y_{1}, y_{2} \in Y$,

(ii) $C M_{B}\left(f\left(x_{1} x_{2}\right)\right) \geq C M_{B}\left(f\left(x_{1}\right)\right) \wedge C M_{B}\left(f\left(x_{2}\right)\right) \forall x_{1}, x_{2} \in X$, where

(i) the image of $A$ under $f$, denoted by $f(A)$, is a fuzzy multiset over $Y$ defined by

$$
C M_{f(A)}(y)= \begin{cases}\bigvee_{x \in f^{-1}(y)} C M_{A}(x), & f^{-1}(y) \neq \emptyset \\ 0, & \text { otherwise }\end{cases}
$$

for each $y \in Y$.

(ii) the inverse image of $B$ under $f$, denoted by $f^{-1}(B)$, is a fuzzy multiset over $X$ defined by

$$
C M_{f^{-1}(B)}(x)=C M_{B}(f(x)) \forall x \in X .
$$

Theorem 2.12 ([9]). Let $X, Y$ be two groups and $f: X \rightarrow Y$ be a homomorphism. If $A \in F M G(X)$ and $B \in F M G(Y)$, respectively, then $f(A) \in F M G(Y)$ and $f^{-1}(B) \in F M G(X)$. 
Definition 2.13 ([12]). Let $A$ be a fuzzy submultigroup of $B \in F M G(X)$. Then, $A$ is called a characteristic (f-invariant) fuzzy submultigroup of $B$ if

$$
C M_{A^{\theta}}(x)=C M_{A}(x) \forall x \in X
$$

for every automorphism, $\theta$ of $X$. That is, $\theta(A) \subseteq A$ for every $\theta \in A u t(X)$.

It follows from [12] that, every characteristic fuzzy submultigroup of a fuzzy multigroup is normal.

\section{Commutator of fuzzy multigroups}

Recall that the commutator of two elements $x$ and $y$ of a group $X$ is the element $[x, y]=x^{-1} y^{-1} x y \in X$. If $H$ and $K$ are subgroups of $X$, then the commutator subgroup or derived subgroup $[H, K]$ of $X$ is generated by $\{[x, y] \mid x \in H, y \in K\}$. Now, the idea of commutator in fuzzy multigroup context is introduced.

Definition 3.1. Let $A$ and $B$ be fuzzy submultigroups of $C \in F M G(X)$. Then, $(A, B)$ is a fuzzy multiset of $X$ defined as follows: $\forall x \in X$

$$
C M_{(A, B)}(x)= \begin{cases}\bigvee_{x=[a, b]}\left[C M_{A}(a) \wedge C M_{B}(b)\right], & \text { if } x \text { is a commutator in } X \\ 0, & \text { otherwise }\end{cases}
$$

The commutator of $A$ and $B$ is a fuzzy multigroup $[A, B]$ of $X$ generated by $(A, B)$.

Now, we present some properties of commutator in fuzzy multigroup context as follow:

Proposition 3.2. Suppose $A \in F M G(X)$ and $[x, y]$ is a commutator of $x$ and $y$ in $A$. Then $C M_{A}([x, y])=C M_{A}(e)$ if and only if $A$ is commutative.

Proof. Assume $C M_{A}([x, y])=C M_{A}(e)$, where $e$ is the identity element in $A$. Then $C M_{A}\left(x^{-1} y^{-1} x y\right)=C M_{A}(e) \Rightarrow C M_{A}\left((y x)^{-1} x y\right)=C M_{A}(e) \Rightarrow C M_{A}(x y)=$ $C M_{A}(y x)$. Thus, $A$ is commutative. 
Conversely, if $C M_{A}(x y)=C M_{A}(y x)$. Then, we have

$$
\begin{aligned}
C M_{A}\left((y x)^{-1} x y\right) & =C M_{A}\left((y x)^{-1} y x\right) \\
& =C M_{A}\left(\left(x^{-1} x\right)\left(y^{-1} y\right)\right) \\
& \geq C M_{A}\left(x^{-1} x\right) \wedge C M_{A}\left(y^{-1} y\right) \\
& =C M_{A}(e),
\end{aligned}
$$

Thus, $C M_{A}\left(x^{-1} y^{-1} x y\right)=C M_{A}([x, y]) \geq C M_{A}(e)$. Again, we have $C M_{A}(e)=$ $C M_{A}\left((y x)^{-1} y x\right) \geq C M_{A}([x, y])$, since $C M_{A}(e) \geq C M_{A}(x) \forall x \in X$. Thus, $C M_{A}([x, y])=C M_{A}(e)$. The result follows.

Proposition 3.3. Suppose $A \in F M G(X)$. If $A$ is commutative, then $C M_{A}\left([x, y]^{-1}\right)=C M_{A}([y, x]) \forall x, y \in X$.

Proof. Suppose $A$ is commutative. Then, we get

$$
\begin{aligned}
C M_{A}\left([x, y]^{-1}\right) \geq C M_{A}([x, y]) & =C M_{A}\left(x^{-1} y^{-1} x y\right) \\
& =C M_{A}\left(y^{-1} x^{-1} y x\right) \\
& =C M_{A}([y, x]),
\end{aligned}
$$

$\Rightarrow C M_{A}\left([x, y]^{-1}\right) \geq C M_{A}([y, x])$. Similarly,

$$
\begin{aligned}
C M_{A}([y, x])=C M_{A}\left(\left([y, x]^{-1}\right)^{-1}\right) & \geq C M_{A}\left([y, x]^{-1}\right) \\
& =C M_{A}\left(\left(y^{-1} x^{-1} y x\right)^{-1}\right) \\
& =C M_{A}\left(\left(x^{-1} y^{-1} x y\right)^{-1}\right) \\
& =C M_{A}\left([x, y]^{-1}\right),
\end{aligned}
$$

$\Rightarrow C M_{A}([y, x]) \geq C M_{A}\left([x, y]^{-1}\right)$. Hence, $C M_{A}\left([x, y]^{-1}\right)=C M_{A}([y, x]) \forall x, y \in$ $X$.

Remark 3.4. If a fuzzy multigroup $A$ of $X$ is commutative, then it is easy to see that $C M_{A}\left(z[x, y] z^{-1}\right)=C M_{A}\left(\left[z x z^{-1}, z y z^{-1}\right]\right) \forall x, y, z \in X$.

Proposition 3.5. Let $x, y, z \in X$ and $A$ be a commutative fuzzy multigroup of $X$. Then 
(i) $C M_{A}([x y, z])=C M_{A}\left([x, z]^{y}[y, z]\right)$,

(ii) $C M_{A}([x, y z])=C M_{A}\left([x, z][x, y]^{z}\right)$.

Proof. For $x, y, z \in X$, we have

(i)

$$
\begin{aligned}
C M_{A}([x y, z]) & =C M_{A}\left((x y)^{-1} z^{-1} x y z\right) \\
& =C M_{A}\left(y^{-1} x^{-1} z^{-1} x y z\right) \\
& =C M_{A}\left(y^{-1} x^{-1} z^{-1} x z y\right) \\
& =C M_{A}\left([x, z]^{y}\right) .
\end{aligned}
$$

Similarly,

$$
\begin{aligned}
C M_{A}\left([x, y]^{y}[y, z]\right) & =C M_{A}\left(\left(y^{-1} x^{-1} y^{-1} x y y\right)[y, z]\right) \\
& =C M_{A}\left([x, z]^{y}\right) .
\end{aligned}
$$

Hence, the result.

(ii)

$$
\begin{aligned}
C M_{A}([x, y z]) & =C M_{A}\left(x^{-1}(y z)^{-1} x y z\right) \\
& =C M_{A}\left(x^{-1} z^{-1} y^{-1} x y z\right) \\
& =C M_{A}\left([x, y]^{z}\right)
\end{aligned}
$$

and similarly, we have

$$
\begin{aligned}
C M_{A}\left([x, z][x, y]^{z}\right) & =C M_{A}\left(x^{-1} z^{-1} y^{-1} x y z\right) \\
& =C M_{A}\left([x, y]^{z}\right) .
\end{aligned}
$$

The result follows.

Lemma 3.6. If $x, y, z \in X$ and $A$ is a commutative fuzzy multigroup of $X$, we have 
(i) $C M_{A}([x y, z])=C M_{A}([x, z][x, z, y][y, z])$,

(ii) $C M_{A}([x, y z])=C M_{A}([x, z][x, y][x, y, z])$.

Proof. Straightforward from Proposition 3.5 .

Proposition 3.7. Let $x, y, z \in X$, and $A$ be a fuzzy multigroup of $X$. Then, we have $C M_{A}\left(\left[x, y^{-1}, z\right]^{y}\left[y, z^{-1}, x\right]^{z}\left[z, x^{-1}, y\right]^{x}\right)=C M_{A}(e)$, where $e$ is the identity of $X$.

Proof. For $x, y, z \in X$, we have

$$
\begin{aligned}
C M_{A}\left(\left[x, y^{-1}, z\right]^{y}\right) & =C M_{A}\left(y^{-1}\left[x^{-1} y x y^{-1}, z\right] y\right) \\
& =C M_{A}\left(y^{-1}\left(y x^{-1} y^{-1} x\right) z^{-1}\left(x^{-1} y x y^{-1}\right) z y\right) \\
& =C M_{A}\left(x^{-1} y^{-1} x z^{-1} x^{-1} y x y^{-1} z y\right) .
\end{aligned}
$$

Setting $a=x z x^{-1} y x, b=y x y^{-1} z y$ and $c=z y z^{-1} x z$. By observation, $b$ and $c$ can be obtained by cyclic permutation of $x, y, z$. Furthermore, we see that $C M_{A}\left(\left[x, y^{-1}, z\right]^{y}\right)=C M_{A}\left(a^{-1} b\right)$. Deducibly, it follows that $C M_{A}\left(\left[y, z^{-1}, x\right]^{z}\right)=$ $C M_{A}\left(b^{-1} c\right)$ and $C M_{A}\left(\left[z, x^{-1}, y\right]^{x}\right)=C M_{A}\left(c^{-1} a\right)$. Since

$$
C M_{A}\left(\left(a^{-1} b\right)\left(b^{-1} c\right)\left(c^{-1} a\right)\right)=C M_{A}(e),
$$

the result follows.

Theorem 3.8. Let $x, y \in X, z=[x, y]$ commutes with both $x$ and $y$. If $A$ is a fuzzy multigroup of $X$, then $C M_{A}\left(\left[x^{i}, y^{j}\right]\right)=C M_{A}\left(z^{i j}\right) \forall i, j$.

Proof. By a given hypothesis, $C M_{A}(z)=C M_{A}\left(x^{-1} y^{-1} x y\right)$, and so $C M_{A}\left(y^{-1} x y\right)=C M_{A}(x z)$, where

$$
\begin{aligned}
C M_{A}\left(y^{-1} x^{i} y\right)=C M_{A}\left(\left(y^{-1} x y\right)^{i}\right) & =C M_{A}\left((x z)^{i}\right) \\
& =C M_{A}\left(x^{i} z^{i}\right)
\end{aligned}
$$

as $x$ and $z$ commute. Conjugating by $y$ gives

$$
\begin{aligned}
C M_{A}\left(y^{-2} x^{i} y^{2}\right)=C M_{A}\left(y^{-1} x^{i} z^{i} y\right) & =C M_{A}\left(y^{-1} x^{i} y z^{i}\right) \\
& =C M_{A}\left(\left(x^{i} z^{i}\right) z^{i}\right) \\
& =C M_{A}\left(x^{i} z^{2 i}\right)
\end{aligned}
$$


as $y$ and $z$ commute. Repeating this argument $j$ times, we have $C M_{A}\left(y^{-j} x^{i} y^{j}\right)=$ $C M_{A}\left(x^{i} z^{i j}\right)$, hence $C M_{A}\left(\left[x^{i}, y^{j}\right]\right)=C M_{A}\left(z^{i j}\right)$.

Theorem 3.9. Let $A \in F M G(X)$ and $x, y, z \in X$. If $y$ commutes with $z$ in $A$ and $X$ is abelian, then $C M_{A}([x, y, z])=C M_{A}([x, z, y)$.

Proof. To start with, we have

$$
\begin{aligned}
C M_{A}([x, y, z]) & =C M_{A}([[x, y], z]) \\
& =C M_{A}\left([x, y]^{-1} z^{-1}[x, y] z\right) \\
& =C M_{A}\left(y^{-1} x^{-1} y x z^{-1} x^{-1} y^{-1} x y z\right) \\
& =C M_{A}\left(x^{-1}\left(x y^{-1} x^{-1} y\right)\left(x z^{-1} x^{-1} z\right) z^{-1} y^{-1} x y z\right) .
\end{aligned}
$$

We observe that $x y^{-1} x^{-1} y$ and $x z^{-1} x^{-1} z$ lies in $X$, and thus commute. It follows that

$$
C M_{A}([x, y, z])=C M_{A}\left(x^{-1}\left(x z^{-1} x^{-1} z\right)\left(x y^{-1} x^{-1} y\right) z^{-1} y^{-1} x y z\right) .
$$

Since $y$ and $z$ commute, then

$$
\begin{aligned}
C M_{A}([x, y, z]) & =C M_{A}\left(z^{-1} x^{-1} z x y^{-1} x^{-1} z^{-1} x z y\right) \\
& =C M_{A}([x, z, y]) .
\end{aligned}
$$

This completes the proof.

Theorem 3.10. Let $A \in F M G(X)$ and $x, y, z \in X$. If $[x, y]$ commutes with both $x$ and $y$, then

$$
C M_{A}\left([x, y]^{-1}\right)=C M_{A}\left(\left[x^{-1}, y\right]\right)=C M_{A}\left(\left[x, y^{-1}\right]\right) .
$$

Proof. By synthesizing Lemma 3.6, we have

$$
C M_{A}(e)=C M_{A}\left(\left[x x^{-1}, y\right]\right)=C M_{A}\left([x, y]\left[x, y, x^{-1}\right]\left[x^{-1}, y\right]\right) .
$$

But, $\left[x, y, x^{-1}\right]=\left[[x, y], x^{-1}\right]$ and $[x, y]$ commutes with $x$ by hypothesis. It follows that $C M_{A}\left(\left[x, y, x^{-1}\right]\right)=C M_{A}(e) \Rightarrow\left[x, y, x^{-1}\right]=e$, where $e$ is the identity of $X$. Thus,

$$
C M_{A}(e)=C M_{A}\left([x, y]\left[x^{-1}, y\right]\right) \Rightarrow C M_{A}\left([x, y]^{-1}\right)=C M_{A}\left(\left[x^{-1}, y\right]\right) .
$$

Similarly, we have $C M_{A}\left([x, y]^{-1}\right)=C M_{A}\left(\left[x, y^{-1}\right]\right)$. Hence, the equality holds. 
Definition 3.11. Let $A, B \in F M G(X)$ such that $A \subseteq B$. Define $A^{(0)}=A$. For $n \in \mathbb{N}$ and suppose $A^{(n-1)}$ can be defined for $n \geq 1$. Then, $A^{(n)}$ can be defined by

$$
A^{(n)}=\left(A^{(n-1)}, A^{(n-1)}\right) .
$$

Theorem 3.12. If $A$ is a fuzzy multigroup of $X$, then $A^{(n)} \subseteq A^{(n-1)}$ for all $n \in \mathbb{N}$.

Proof. We establish the proof by induction on $n$. Let $x \in X$. If $x$ is not a commutator in $X$. Then

$$
C M_{(A, A)}=0 \text { and } C M_{A}(x) \geq 0 \Rightarrow C M_{(A, A)}=0 \leq C M_{A}(x) .
$$

Suppose $x$ is a commutator in $X$. Then, for $a, b \in X$ we have

$$
\begin{aligned}
C M_{(A, A)} & =\bigvee_{x=[a, b]}\left[C M_{A}(a) \wedge C M_{A}(b)\right] \\
& =\bigvee_{x=[a, b]}\left[C M_{A}\left(a^{-1}\right) \wedge C M_{A}\left(b^{-1}\right) \wedge C M_{A}(a) \wedge C M_{A}(b)\right] \\
& \leq \bigvee_{x=[a, b]}\left[C M_{A}\left(a^{-1} b^{-1} a b\right)\right] \\
& =C M_{A}(x)
\end{aligned}
$$

and so $(A, A) \subseteq A$. Since $A \in F M G(X)$, then

$$
C M_{A^{(1)}}(x)=C M_{\left(A^{(0)}, A^{(0)}\right)}(x) \leq C M_{A}(x)=C M_{A^{(0)}}(x),
$$

and so the result follows for $k=1$. Since $C M_{A^{(n)}}(x) \leq C M_{A^{(n-1)}}(x)$ for $n \in \mathbb{N}$, we have

$$
\begin{aligned}
C M_{A^{(k+1)}}(x) & =C M_{\left(A^{(k)}, A^{(k)}\right)}(x) \\
& \leq C M_{\left(A^{(k-1)}, A^{(k-1)}\right)}(x) \\
& =C M_{A^{k}}(x) .
\end{aligned}
$$

Thus, $A^{(k+1)} \subseteq A^{(k)} \Rightarrow A^{(k)} \subseteq A^{(k-1)}$. Hence, the result follows for $n=k$. 
Theorem 3.13. If $A$ and $B$ are normal (characteristic, fully invariant) fuzzy submultigroups of a fuzzy multigroup $C$ of $X$. Then $[A, B]$ is a normal (characteristic, fully invariant) fuzzy submultigroup of $C$ contained in $A \cap B$.

Proof. Suppose $A$ and $B$ are normal (characteristic, fully invariant) fuzzy submultigroups of $C$, then $[A, B]$ is a normal (characteristic, fully invariant) fuzzy submultigroups of $C$. Because $A$ and $B$ are normal and so, they assume constant value on the conjugacy classes of $X$.

Since $A \cap B$ is a fuzzy multigroup of $X$, it suffices to proves that $(A, B) \subseteq A \cap B$. Let $x \in X$. If $x$ is not a commutator, then

$$
C M_{(A, B)}(x)=0 \leq C M_{A \cap B}(x) .
$$

Suppose that $x=a b a^{-1} b^{-1}$ for some $a, b \in X$. Then

$$
\begin{aligned}
C M_{A \cap B}(x) & =C M_{A}(x) \wedge C M_{B}(x) \\
& =C M_{A}\left(a b a^{-1} b^{-1}\right) \wedge C M_{B}\left(a b a^{-1} b^{-1}\right) \\
& \geq\left[C M_{A}(a) \wedge C M_{A}\left(b a^{-1} b^{-1}\right)\right] \wedge\left[C M_{B}\left(a b a^{-1}\right) \wedge C M_{B}\left(b^{-1}\right)\right] \\
& \geq\left[C M_{A}(a) \wedge C M_{A}\left(a^{-1}\right)\right] \wedge\left[C M_{B}(b) \wedge C M_{B}\left(b^{-1}\right)\right] \\
& =C M_{A}(a) \wedge C M_{B}(b) .
\end{aligned}
$$

This implies that

$$
\begin{aligned}
C M_{A \cap B}(x) & \geq \bigvee_{\substack{x=a b a^{-1} b^{-1}\\
}} C M_{A}(a) \wedge C M_{B}(b) \\
& =C M_{(A, B)}(x) .
\end{aligned}
$$

Thus, $(A, B) \subseteq A \cap B$. Consequently, $[A, B] \subseteq A \cap B$.

Corollary 3.14. Using the same hypothesis in Theorem 3.13, then $[A, B]$ is a normal (characteristic, fully invariant) fuzzy submultigroup of $C$ contained in $A \cup B$.

Proof. By Definition 2.2, we get $A \cap B \subseteq A \cup B$. Hence, the result follows by transitivity because $[A, B] \subseteq A \cap B$. 
Theorem 3.15. Suppose $A$ and $B$ are fuzzy submultigroups of $C \in F M G(X)$. Then $[A, B]=[B, A]$.

Proof. Let $x \in X$. Since $[A, B]$ is a fuzzy multigroup generated by $(A, B)$, if we prove that $(A, B)=(B, A)$ we are done. Assume $x$ is not a commutator in $X$, then $x^{-1}$ is not a commutator and consequently,

$$
C M_{(A, B)}(x)=0=C M_{(B, A)}\left(x^{-1}\right) .
$$

Suppose $x=[a, b]$ for some $a, b \in X$. Then

$$
\begin{aligned}
C M_{(A, B)}(x) & =\bigvee_{x=[a, b]}\left[C M_{A}(a) \wedge C M_{B}(b)\right] \\
& =\bigvee_{x^{-1}=[b, a]}\left[C M_{B}(b) \wedge C M_{A}(a)\right] \\
& =C M_{(B, A)}\left(x^{-1}\right) .
\end{aligned}
$$

Hence, $(A, B)=(B, A)$ and the result follows.

Corollary 3.16. If $A$ and $B$ are fuzzy submultisets of $C \in F M G(X)$. Then

(i) $[A, B]_{*}=[B, A]_{*}$,

(ii) $[A, B]^{*}=[B, A]^{*}$.

Proof. Using Proposition 2.7 and Theorem 3.15, the results follow.

Corollary 3.17. If $A$ and $B$ are fuzzy submultisets of $C \in F M G(X)$. Then, for $\alpha \in[0,1]$,

(i) $[A, B]_{[\alpha]}=[B, A]_{[\alpha]}$,

(ii) $[A, B]^{[\alpha]}=[B, A]^{[\alpha]}$.

Proof. Combining Proposition 2.8 and Theorem 3.15, the results follow.

Theorem 3.18. If $A \in F M G(X)$, then $[A, A] \subseteq A$. 
Proof. If $x$ is not a commutator in $X$, then $C M_{(A, A)}(x)=0$. Suppose $x$ is a commutator, then

$$
\begin{aligned}
C M_{(A, A)}(x) & =\bigvee_{x=[a, b]}\left[C M_{A}(a) \wedge C M_{A}(b)\right] \text { for some } a, b \in X \\
& =\bigvee_{x=[a, b]}\left[C M_{A}\left(a^{-1}\right) \wedge C M_{A}\left(b^{-1}\right) \wedge C M_{A}(a) \wedge C M_{A}(b)\right] \\
& \leq \bigvee_{x=[a, b]}\left[C M_{A}\left(a^{-1} b^{-1} a b\right)\right] \\
& =C M_{A}(x) .
\end{aligned}
$$

Thus, $(A, A) \subseteq A \Rightarrow[A, A] \subseteq A$.

Theorem 3.19. If $A, B, C, D \in F M G(X)$ such that $A \subseteq B$ and $C \subseteq D$, then $[A, C] \subseteq[B, D]$.

Proof. Recall that $[A, C]=\langle(A, C)\rangle$. If $x$ is not a commutator in $X$, then $C M_{(A, B)}(x)=0$ and therefore there is nothing to prove. Suppose $x$ is a commutator, then

$$
\begin{aligned}
C M_{(A, C)}(x) & =\bigvee_{x=[a, b]}\left[C M_{A}(a) \wedge C M_{C}(b)\right] \text { for some } a, b \in X \\
& \leq \bigvee_{x=[a, b]}\left[C M_{B}(a) \wedge C M_{D}(b)\right] \\
& =C M_{(B, D)}(x) .
\end{aligned}
$$

Thus, $[A, C]=\langle(A, C)\rangle \subseteq\langle(B, D)\rangle=[B, D]$. Hence, $[A, C] \subseteq[B, D]$.

Corollary 3.20. Let $A, B, C, D, E, F \in F M G(X)$. If $[C, D] \subseteq[A, B]$ and $[E, F] \subseteq[A, B]$, then $[[C, D],[E, F]] \subseteq[A, B]$.

Proof. Suppose $[C, D] \subseteq[A, B]$ and $[E, F] \subseteq[A, B]$. Then $[[C, D],[E, F]] \subseteq$ $[[A, B],[A, B]] \subseteq[A, B]$. Hence, $[[C, D],[E, F]] \subseteq[A, B]$.

Proposition 3.21. Let $A, B \in F M G(X)$. Then $[A, B] \circ[B, A]=[A, B]$. 
Proof. Combining Proposition 2.5 and Theorem 3.15 , we get $[A, B] \circ[B, A] \subseteq$ $[A, B]$. Again, if $x=0$, we have $C M_{[A, B] \circ[B, A]}(x)=0$. Otherwise,

$$
\begin{aligned}
C M_{[A, B] \circ[B, A]}(x) & =\bigvee_{x=a b}\left[C M_{[A, B]}(a) \wedge C M_{[B, A]}(b)\right] \\
& \geq C M_{[A, B]}(x) \wedge C M_{[A, B]}(e) \\
& =C M_{[A, B]}(x),
\end{aligned}
$$

and so, $[A, B] \subseteq[A, B] \circ[B, A]$. Hence, $[A, B] \circ[B, A]=[A, B]$.

Proposition 3.22. Let $A, B, C, D, E, F \in F M G(X)$ such that $[C, D] \subseteq[A, B]$ and $[E, F] \subseteq[A, B]$. Then $[C, D] \circ[E, F] \subseteq[A, B]$.

Proof. Let $x \in X$. If $x=0$, we have $C M_{[C, D] \circ[E, F]}(x)=0$. Otherwise,

$$
\begin{aligned}
C M_{[C, D] \circ[E, F]}(x) & =\bigvee_{x=a b}\left[C M_{[C, D]}(a) \wedge C M_{[E, F]}(b)\right] \\
& \leq \bigvee_{x=a b}\left[C M_{[A, B]}(a) \wedge C M_{[A, B]}(b)\right] \\
& =C M_{[A, B] \circ[A, B]}(x) \\
& =C M_{[A, B]}(x) .
\end{aligned}
$$

Hence, $[C, D] \circ[E, F] \subseteq[A, B]$.

Lemma 3.23. Suppose $A$ and $B$ are fuzzy submultigroups of $D \in F M G(X)$ such that $A \subseteq B$, then $[A, C] \subseteq[B, C]$ for any fuzzy submultigroup $C$ of $D$.

Proof. Given $A \subseteq B$, then $C M_{A}(x) \leq C M_{B}(x) \forall x \in X$. Let $C$ be any fuzzy submultigroup of $D$. If $x$ is not a commutator in $X$, then

$$
C M_{(A, C)}(x)=0=C M_{(B, C)}(x) .
$$

Suppose $x=[a, b]$ for some $a, b \in X$. Then, we have

$$
\begin{aligned}
C M_{(A, C)}(x) & =\bigvee_{x=[a, b]}\left[C M_{A}(a) \wedge C M_{C}(b)\right] \\
& \leq \bigvee_{x=[a, b]}\left[C M_{B}(a) \wedge C M_{C}(b)\right] \\
& =C M_{(B, C)}(x),
\end{aligned}
$$


implies that $(A, C) \subseteq(B, C)$ and so $[A, C] \subseteq[B, C]$.

Theorem 3.24. Let $A$ and $B$ be normal fuzzy submultigroups of $D \in F M G(X)$ and let $C$ be any fuzzy submultigroup of $D$. Then $[A \circ C, B] \subseteq[A, B] \circ[C, B]$ with equality holding if $C M_{A}(e)=C M_{C}(e)$, where $e$ is the identity of $X$.

Proof. Firstly, we show that $(A \circ C, B) \subseteq[A, B] \circ[C, B]$. Let $x \in X$. If $x$ is not a commutator in $X$, then $C M_{(A \circ C, B)}(x)=0$ and the result is trivial. Suppose $x$ is a commutator in $X$. Then

$$
\begin{aligned}
C M_{(A \circ C, B)}(x) & =\bigvee\left\{C M_{A \circ C}(a) \wedge C M_{B}(b) \mid x=[a, b], a, b \in X\right\} \\
& =\bigvee\left\{\bigvee\left\{C M_{A}(u) \wedge C M_{C}(v) \mid a=u v, u, v \in X\right\} \wedge C M_{B}(b) \mid x=[a, b], a, b \in X\right\} \\
& =\bigvee\left\{\bigvee\left\{\left[C M_{A}(u) \wedge C M_{C}(v)\right] \wedge C M_{B}(b) \mid a=u v, u, v \in X\right\} \mid x=[a, b], a, b \in X\right\} \\
& =\bigvee\left\{\bigvee\left\{\left[C M_{A}(u) \wedge C M_{B}(b)\right] \wedge\left[C M_{C}(v) \wedge C M_{B}(b) \mid a=u v, u, v \in X\right\} \mid x=[a, b], a, b \in X\right\}\right. \\
& =\bigvee\left\{\left[C M_{A}(u) \wedge C M_{B}(b)\right] \wedge\left[C M_{C}(v) \wedge C M_{B}(b)\right] \mid x=[u v, b], u v, b \in X\right\} \\
& \leq \bigvee\left\{C M_{[A, B]}([u, b]) \wedge C M_{[C, B]}([v, b]) \mid x=[u v, b], u v, b \in X\right\} \\
& \left.=\bigvee\left\{C M_{[A, B]}([u, b]]^{v}\right) \wedge C M_{[C, B]}([v, b]) \mid x=[u v, b], u v, b \in X\right\} \\
& \leq \bigvee\left\{C M_{[A, B]}(y) \wedge C M_{[C, B]}(z) \mid x=y z, y, z \in X\right\} \\
& =C M_{[A, B] \circ[C, B]}(x),
\end{aligned}
$$

where $y=[u, b]^{v}, z=[v, b]$ and $x=y z=[u v, b]=[u, b]^{v}[v, b]$. Since $[A, B]$ is normal, $[A, B] \circ[C, B]$ is a fuzzy multigroup of $X$. Hence, $[A \circ C, B] \subseteq[A, B] \circ[C, B]$.

Finally, suppose $C M_{A}(e)=C M_{C}(e)$. Then, $A \subseteq A \circ C$ and $C \subseteq A \circ C$. Thus, $[A, B] \subseteq[A \circ C, B]$ and $[C, B] \subseteq[A \circ C, B]$ by Lemma 3.23 . Hence, $[A, B] \circ[C, B] \subseteq$ $[A \circ C, B]$ since $[A \circ C, B]$ is a fuzzy multigroup of $X$. Consequently, the desired equality holds.

\section{Some homomorphic properties of commutator in fuzzy multigroup context}

Now, we consider homomorphic images and preimages of commutator of fuzzy multigroups.

Lemma 4.1. Let $f$ be a homomorphism of a group $X$ into a group $Y$. If $A$ and $B$ are fuzzy submultisets of $C \in F M G(X)$, then $f([A, B])$ is a fuzzy multigroup of $Y$ generated by $f((A, B))$. 
Proof. Since $[A, B]$ is generated by $(A, B)$, then $f([A, B])$ is a fuzzy multigroup of $Y$ generated by $f((A, B))$, which is a direct consequent of Theorem 2.12 .

Lemma 4.2. Let $f$ be an isomorphism of a group $X$ into a group $Y$. If $A$ and $B$ are fuzzy submultisets of $C \in F M G(Y)$, then $f^{-1}([A, B])$ is a fuzzy multigroup of $X$ generated by $f^{-1}((A, B))$.

Proof. Similar to Lemma 4.1 .

Theorem 4.3. If $f$ is a homomorphism of a group $X$ into a group $Y$. Then $[f(A), f(B)]=f([A, B])$ for fuzzy multigroups $A$ and $B$ of $X$.

Proof. Foremostly, we show that $f((A, B)) \subseteq[f(A), f(B)]$. Let $y \in Y$. If $f^{-1}(y)=\emptyset$, then

$$
C M_{f(A, B)}(y)=0 \leq C M_{[f(A), f(B)]}(y) .
$$

Assume $y=f(x)$ for some $x \in X$. Then, we have

$$
\begin{aligned}
C M_{(A, B)}(x) & =\bigvee\left\{C M_{A}(a) \wedge C M_{B}(b) \mid x=[a, b], a, b \in X\right\} \\
& \leq \bigvee\left\{C M_{f(A)}(f(a)) \wedge C M_{f(B)}(f(b)) \mid y=[f(a), f(b)], a, b \in X\right\} \\
& \leq \bigvee\left\{C M_{f(A)}(c) \wedge C M_{f(B)}(d) \mid y=[c, d], c, d \in X\right\} \\
& =C_{(f(A), f(B))}(y) \\
& \leq C_{[f(A), f(B)]}(y) .
\end{aligned}
$$

Hence,

$$
\begin{aligned}
C M_{(f(A, B))}(y) & =\bigvee\left\{C M_{(A, B)}(x) \mid y=f(x), x \in X\right\} \\
& \leq C M_{[f(A), f(B)]}(y) .
\end{aligned}
$$

Thus, $f((A, B)) \subseteq[f(A), f(B)]$. By Lemma 4.1, $f([A, B]) \subseteq[f(A), f(B)]$.

Now, we proof that $(f(A), f(B)) \subseteq f([A, B])$. Let $y \in Y$. If $y$ is not a commutator of $Y$, then

$$
C M_{(f(A), f(B))}(y)=0 \leq C M_{f([A, B])}(y) .
$$


Suppose $y$ is a commutator in $Y$. Then, $y=[u, v]$ for some $u, v \in Y$. If either $f^{-1}(u)=\emptyset$ or $f^{-1}(v)=\emptyset$, then

$$
C M_{f(A)}(u) \wedge C M_{f(B)}(v)=0 .
$$

Otherwise, we get

$$
\begin{aligned}
C M_{f(A)}(u) \wedge C M_{f(B)}(v) & =\left(\bigvee\left\{C M_{A}(s) \mid u=f(s)\right\}\right) \wedge\left(\bigvee\left\{C M_{B}(t) \mid v=f(t)\right\}\right) \\
& =\bigvee\left\{C M_{A}(s) \wedge C M_{B}(t) \mid u=f(s), v=f(t)\right\} \\
& \leq \bigvee\left\{C M_{(A, B)}([s, t]) \mid y=f([s, t])\right\} \\
& \leq \bigvee\left\{C M_{[A, B]}([s, t]) \mid y=f([s, t])\right\} \\
& \leq \bigvee\left\{C M_{[A, B]}(x) \mid y=f(x)\right\} \\
& =C M_{f([A, B])}(y) .
\end{aligned}
$$

Thus, $(f(A), f(B)) \subseteq f([A, B]) \Rightarrow[f(A), f(B)] \subseteq f([A, B])$. Hence, the equality holds.

Theorem 4.4. Let $f: X \rightarrow Y$ be a homomorphism such that $A, B \in F M G(X)$. Then $\left[f^{-1}(A), f^{-1}(B)\right] \subseteq f^{-1}([A, B])$ in $F M G(X)$.

Proof. Let $x \in X$. If $x$ is not a commutator in $X$, then

$$
C M_{\left(f^{-1}(A), f^{-1}(B)\right)}(x)=0 \leq C M_{\left[f^{-1}(A), f^{-1}(B)\right]}(x) .
$$

Assume that $x$ is a commutator in $X$. Then, we have

$$
\begin{aligned}
C M_{\left(f^{-1}(A), f^{-1}(B)\right)}(x) & =\bigvee\left\{C M_{A}(f(a)) \wedge C M_{B}(f(b)) \mid x=[a, b], a, b \in X\right\} \\
& =\bigvee\left\{C M_{A}(f(a)) \wedge C M_{B}(f(b)) \mid f(x)=[f(a), f(b)], a, b \in X\right\} \\
& \leq \bigvee\left\{C M_{A}(c) \wedge C M_{B}(d) \mid f(x)=[c, d], c, d \in X\right\} \\
& =C M_{(A, B)}(f(x)) \\
& \leq C M_{[A, B]}(f(x)) .
\end{aligned}
$$

Hence, $\left(f^{-1}(A), f^{-1}(B)\right) \subseteq f^{-1}([A, B])$ and consequently, $\left[f^{-1}(A), f^{-1}(B)\right] \subseteq$ $f^{-1}([A, B])$. 
Definition 4.5. A non-empty collection $\mathcal{D}$ of endomorphisms of a group $X$ is an operator domain on $X$. A fuzzy multiset $A$ of $X$ is admissible under $\mathcal{D}$ or $\mathcal{D}$-admissible if for every $f \in \mathcal{D}, f(A) \subseteq A$, where $f$ is a function. Thus, $f(A) \subseteq A$ if and only if $A \subseteq f^{-1}(A)$.

Theorem 4.6. Suppose $A$ and $B$ are admissible fuzzy submultisets of $C \in$ $F M G(X)$ under an operator domain $\mathcal{D}$, then $(A, B)$ and $[A, B]$ are $\mathcal{D}$-admissible.

Proof. The fact that $A$ and $B$ are $\mathcal{D}$-admissible, we have

$$
C M_{f^{-1}(A)}(x) \geq C M_{A}(x) \text { and } C M_{f^{-1}(B)}(x) \geq C M_{B}(x) \forall f \in \mathcal{D} .
$$

Let $f \in \mathcal{D}$ and $x \in X$. If $x$ is not a commutator in $X$, then

$$
C M_{(A, B)}(x)=0 \leq C M_{(A, B)}(f(x)) .
$$

Suppose $x=[a, b]$ for some $a, b \in X$. Then

$$
\begin{aligned}
C M_{(A, B)}(x) & =\bigvee_{x=[a, b]}\left[C M_{A}(a) \wedge C M_{B}(b)\right] \\
& \leq \bigvee_{x=[a, b]}\left[C M_{A}(f(a)) \wedge C M_{B}(f(b))\right] \\
& =\bigvee_{f(x)=[f(a), f(b)]}\left[C M_{A}(f(a)) \wedge C M_{B}(f(b))\right] \\
& \leq \bigvee_{f(x)=[c, d]}\left[C M_{A}(c) \wedge C M_{B}(d)\right], c, d \in X \\
& =C M_{(A, B)}(f(x)) .
\end{aligned}
$$

Thus, $(A, B) \subseteq f^{-1}((A, B))$. Hence, $(A, B)$ is $\mathcal{D}$-admissible. Since $[A, B]$ is generated by $(A, B)$, it follows that $(A, B) \subseteq f^{-1}([A, B])$. Thus, $[A, B] \subseteq$ $f^{-1}([A, B])$. Hence, $[A, B]$ is $\mathcal{D}$-admissible.

\section{Conclusion}

This work further the study of fuzzy multigroup theory parallel to crisp group theory. The ideas of commutator of fuzzy multigroups and commutator fuzzy 
submultigroups of fuzzy multigroups were proposed and characterized with a number of some related results. Some homomorphic images and preimages of commutator of fuzzy multigroups were considered with some results. The notion of admissible fuzzy submultisets $A$ and $B$ of $C \in F M G(X)$ under an operator domain $\mathcal{D}$ was explicated, and it was shown that $(A, B)$ and $[A, B]$ are $\mathcal{D}$-admissible. However, more properties of commutator in fuzzy multigroup setting could be exploited in future investigation.

\section{References}

[1] N. Ajmal and A.S. Prajapati, Fuzzy cosets and fuzzy normal subgroups, Inform. Sci. 64 (1992), 17-25. https://doi.org/10.1016/0020-0255(92)90107-J

[2] A. Baby, T.K. Shinoj and J.J. Sunil, On abelian fuzzy multigroups and order of fuzzy multigroups, J. New Theory 5(2) (2015), 80-93.

[3] R. Biswas, An application of Yager's bag theory in multicriteria based decision making problems, Int. J. Intell. Syst. 14 (1999), 1231-1238. https://doi.org/10.1002/(SICI) 1098-111X(199912)14:12\%3C1231:: AID-INT4\%3E3.0.CD;2-6

[4] P. Bhattacharya and N.P. Mukherjee, Fuzzy groups: some group theoretic analogs II, Inform. Sci. 41 (1987), 77-91. https://doi.org/10.1016/0020-0255(87)90006-5

[5] P.A. Ejegwa, Correspondence between fuzzy multisets and sequences, Global J. Sci. Frontier Research: Math. Decision Sci. 14(7) (2014), 61-66.

[6] P.A. Ejegwa, On abelian fuzzy multigroups, J. Fuzzy Math. 26(3) (2018), 655-668.

[7] P.A. Ejegwa, On fuzzy multigroups and fuzzy submultigroups, J. Fuzzy Math. 26(3) (2018), 641-654.

[8] P.A. Ejegwa, On normal fuzzy submultigroups of a fuzzy multigroup, Theory Appl. Math. Comp. Sci. 8(1) (2018), 64-80. 
[9] P.A. Ejegwa, Homomorphism of fuzzy multigroups and some of its properties, Appl. Appli. Math. 13(1) (2018), 114-129.

[10] P.A. Ejegwa, Direct product of fuzzy multigroups, J. New Theory 28 (2019), 62-73.

[11] P.A. Ejegwa, Synopsis of the notions of multisets and fuzzy multisets, Ann. Commun. Math. 2(2) (2019), 101-120.

[12] P.A. Ejegwa, Some group's theoretic notions in fuzzy multigroup context, in: Handbook of Research on Emerging Applications of Fuzzy Algebraic Structures, IGI Global Publisher, Hershey, Pennsylvania, USA, 2020, pp. 34-62. https://doi.org/10.4018/978-1-7998-0190-0.ch003

[13] P.A. Ejegwa and J.A. Otuwe, Frattini fuzzy subgroups of fuzzy groups, J. Univ. Math. 2(2) (2019), 175-182.

[14] D. Knuth, The art of computer programming, Semi Numerical Algorithms, 2nd ed., Addison-Wesley, Reading, Massachusetts, 1981.

[15] S. Miyamoto, Basic operations of fuzzy multisets, J. Japan Soc. Fuzzy Theory and Systems 8(4) (1996), 639-645. https://doi.org/10.3156/jfuzzy.8.4_47

[16] S. Miyamoto and K. Mizutani, Fuzzy multiset model and methods for nonlinear document clustering for information retrieval, in: Modeling Decisions for Artificial Intelligence, Springer-Verlag, Berlin, Heidelberg, 2004, pp. 273-283.

https://doi.org/10.1007/978-3-540-27774-3_26

[17] K. Mizutani, R. Inokuchi and S. Miyamoto, Algorithms of nonlinear document clustering based on fuzzy multiset model, Int. J. Intell. Syst. 23 (2008), 176-198. https://doi.org/10.1002/int.20263

[18] J.M. Mordeson, K.R. Bhutani and A. Rosenfeld, Fuzzy Group Theory, Springer-Verlag, Berlin, Heidelberg, 2005.

https://doi.org/10.1007/b12359

[19] N.P. Mukherjee and P. Bhattacharya, Fuzzy groups: some group theoretic analogs, Inform. Sci. 39 (1986), 247-268.

https://doi.org/10.1016/0020-0255(86)90039-3 
[20] N.P. Mukherjee and P. Bhattacharya, Fuzzy normal subgroups and fuzzy cosets, Inform. Sci. 34 (1984), 255-239.

https://doi.org/10.1016/0020-0255(84)90050-1

[21] R. Rasuli, t-norms over fuzzy multigroups, Earthline J. Math. Sci. 3(2) (2020), 207-228. https://doi.org/10.34198/ejms.3220.207228

[22] A. Rosenfeld, Fuzzy subgroups, J. Math. Anal. Appl. 35 (1971), 512-517. https://doi.org/10.1016/0022-247X(71)90199-5

[23] T.K. Shinoj, A. Baby and J.J. Sunil, On some algebraic structures of fuzzy multisets, Ann. Fuzzy Math. Inform. 9(1) (2015), 77-90.

[24] A. Syropoulos, On generalized fuzzy multisets and their use in computation, Iranian J. Fuzzy Systems 9(2) (2012), 113-125.

[25] R.R. Yager, On the theory of bags, Int. J. General Systems 13 (1986), 23-37. https://doi.org/10.1080/03081078608934952

[26] L.A. Zadeh, Fuzzy sets, Inform. Control 8 (1965), 338-353.

https://doi.org/10.1016/S0019-9958(65)90241-X

P. A. Ejegwa

Department of Mathematics/Statistics/Computer Science, University of Agriculture, P.M.B. 2373, Makurdi, Nigeria e-mail: ocholohi@gmail.com

\section{J. M. Agbetayo}

Department of Mathematics/Statistics/Computer Science, University of Agriculture, P.M.B. 2373, Makurdi, Nigeria e-mail: agbetayojohnson@gmail.com

This is an open access article distributed under the terms of the Creative Commons Attribution License (http://creativecommons.org/licenses/by/4.0/), which permits unrestricted, use, distribution and reproduction in any medium, or format for any purpose, even commercially provided the work is properly cited. 